\title{
Voltage Optimization in MV Network with Distributed Generation Using Power Consumption Control in Electrolysis Installations
}

\author{
Paweł Pijarski *(1) and Piotr Kacejko
}

Citation: Pijarski, P.; Kacejko, P. Voltage Optimization in MV Network with Distributed Generation Using Power Consumption Control in Electrolysis Installations. Energies 2021, 14, 993. https://doi.org/ 10.3390/en14040993

Academic Editor: Abdul-Ghani Olabi Received: 10 January 2021

Accepted: 7 February 2021

Published: 14 February 2021

Publisher's Note: MDPI stays neutral with regard to jurisdictional claims in published maps and institutional affiliations.

Copyright: (C) 2021 by the authors. Licensee MDPI, Basel, Switzerland. This article is an open access article distributed under the terms and conditions of the Creative Commons Attribution (CC BY) license (https:/ / creativecommons.org/licenses/by/ $4.0 /)$.
Department of Power Engineering, Faculty of Electrical Engineering and Computer Science, Lublin University of Technology, Nadbystrzycka St. 38D, 20-618 Lublin, Poland; p.kacejko@pollub.pl

* Correspondence: p.pijarski@pollub.pl

Abstract: Connecting a large number of distributed sources to the medium and low voltage grid poses many problems. The most important of these are the voltage changes inside the network, what can be observed when the power flow from these sources towards the HV/MV (High Voltage/Medium Voltage) transformer station. In particular, if the power consumption in nodes of the MV network is small and the distance between the place of installation of the source and the substation is large, increases and changes in voltage may be dangerous for the insulation of the network and burdensome for the consumers connected to it. The solution most frequently used to control voltage increases is the appropriate setting of the controller that affects the on-load tap changer of the MV/HV or even MV/LV (Medium Voltage/Low Voltage) transformer. It is also possible to regulate the reactive power of the sources and, of course, to limit their generated active power (curtailment of generation). The development of energy storage technology has made it possible to introduce consumers into the network, whose power can be controlled in a wide range. The article proposes the concept of an innovative voltage control system in the MV network, whose output values are three groups of parameters: $\mathrm{HV} / \mathrm{MV}$ transformer ratio, reactive power of sources and active power of consumers connected in generation nodes. In the technological sense, it has been assumed that the loads are installations of electrolyzers used to produce "green hydrogen", according to the P2G (Power to Gas) formula. The tests consisting in the execution of several hundred calculation cycles for the IEEE 37 test network, using the Monte Carlo simulation, have shown that the subordination of the hydrogen production process to the objectives of voltage control in the MV network clearly contributes to stabilizing its value, while meeting the technological requirements. The control variables of the proposed control system are the result of the optimization algorithm described in the article, the function of which is the quality of network voltage.

Keywords: voltage control; voltage quality; renewable energy; power to gas; electrolysis; hydrogen

\section{Introduction}

The large number of installations of renewable energy sources, connected to the MV and LV grids, are associated with various problems, which are particularly acute in periods of low power demand. If the power generated by distributed sources corresponded to the power consumed at the points of their connection, then the state of the grid would correspond to the normal state of load. However, the specificity of dispersed generation and the technologies used, is that the correlation between the power they produce and the general power demands is negligible. The power flow in the direction of HV /MV stations results in an effect known as "voltage rise". (or sometimes incorrectly as an "overvoltage"), which is the antonym of the voltage drop. Therefore, the voltage at the connection points of disperse sources, as well as at the connection points of receiving installations in their vicinity, may increase above the limit values. A number of works have been published which consider problems resulting from radical change in the characteristics of distribution 
networks, so far regarded as typical (radial system of operation, unidirectional power flow) [1-5]. The variability of network operating conditions-related to load changes, changes of power generated in distributed sources, changes occurring in the HV network, may result in daily voltage fluctuations in a wide range. Of course, one cannot pass the blame for this voltage discomfort entirely on to dispersed sources, but it is certainly worthwhile to analyse what is their actual role in distorting the voltage profile of MV networks. If, under certain conditions, this role proves to be important, it is necessary to propose technical measures which can compensate for this negative impact. These measures are described in the literature [6,7]. In the research of the authors of the present article, attention has so far been focused on the possibility of using the capacity of dispersed sources to consume (or less frequently generate) reactive power [3]. At the same time, a great progress in energy storage technologies has been observed for several years, with P2G (power to gas) technologies playing the greatest role [8], and alkaline water electrolyzers (AEL) are considered to be the cheapest and easiest to access $[9,10]$. Thus, from the voltage control point of view, another executive element may be the active power loads, for which an increase or decrease in power consumption does not affect the continuity of the technological process of the consumption supplied by them. Such a process is the production of hydrogen in electrolyzers, especially if their construction in a modular form is considered. After making such an assumption, it was possible to propose an innovative system of voltage control in the MV network, in which, apart from the traditional tap control, there are dispersed sources consuming or generating reactive power and flexible loads subordinated also to the process of voltage control-Figure 1.

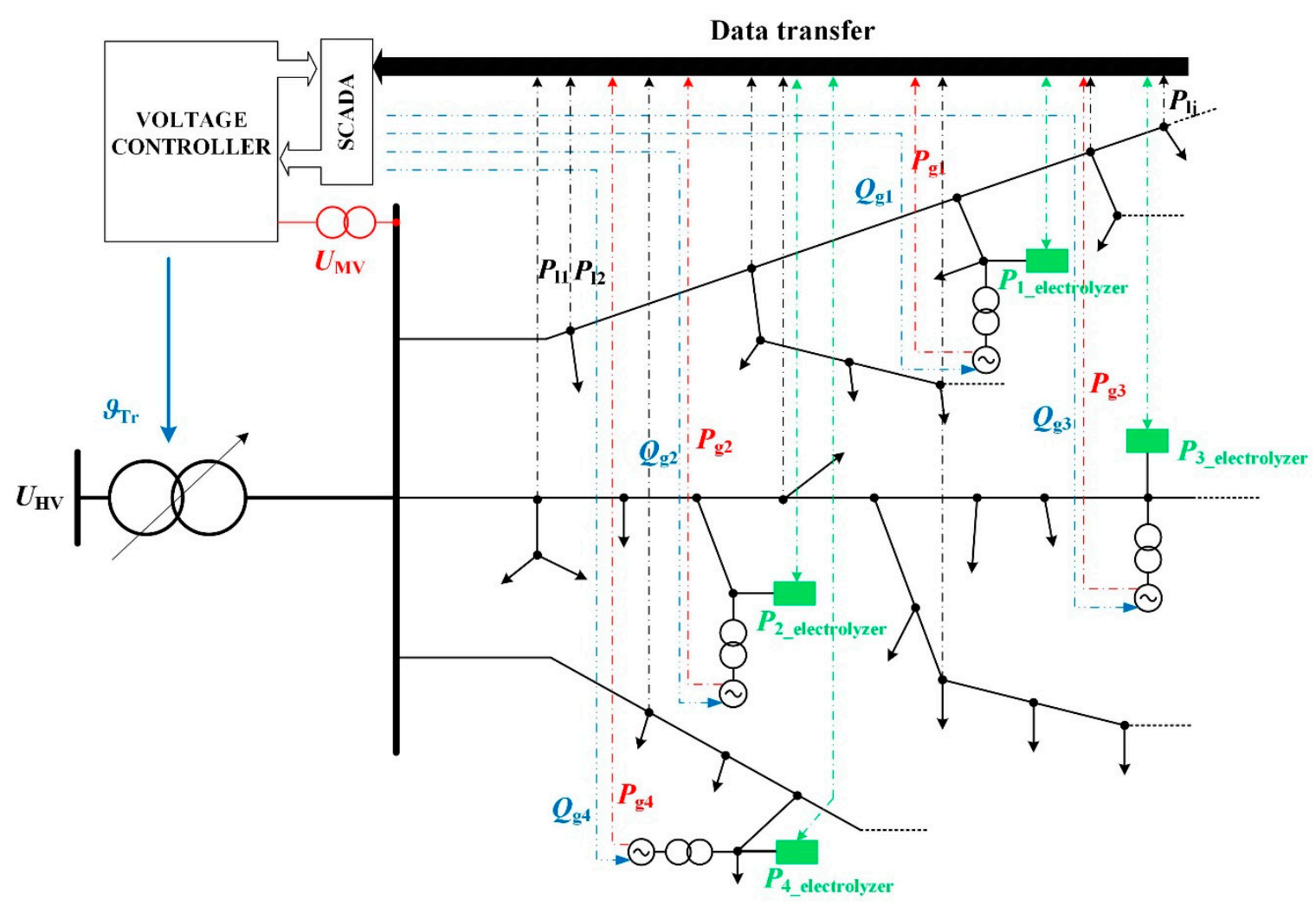

Figure 1. Illustration of the concept of the optimal voltage control system in a MV network with dispersed generation and electrolysis installations $\left(P_{11}, P_{12}, \ldots P_{\mathrm{l} j}\right.$-normal loads, $P_{\mathrm{g} 1}, P_{\mathrm{g} 2}, P_{\mathrm{g} 3}, P_{\mathrm{g} 4}$-active power generation, $Q_{\mathrm{g} 1}, Q_{\mathrm{g} 2}, Q_{\mathrm{g} 3}$, $Q_{\mathrm{g} 4}$-reactive power generation/consumption by RES, $P_{1}, P_{2}, P_{3}, P_{4}$-controllable loads, electrolyzers, $U_{\mathrm{HV}}$ high voltage, $U_{\mathrm{MV}}$ medium voltage, $\vartheta_{\mathrm{Tr}}-\mathrm{HV} / \mathrm{MV}$ transformer ratio).

The present article consists of six chapters. The first chapter contains an introduction to the subject of research and a justification of the advisability of introducing a new method of voltage control using electrolyzers. The second chapter presents a literature review on 
the integration of P2G systems with the power system. The third chapter contains the formulation of the optimization task and a description of the algorithm of its solution. The fourth chapter presents the IEEE 37 test network. The results of calculations showing the effectiveness of the proposed regulation system are included in the fifth chapter. Discussion of the results and conclusions are contained in the sixth chapter.

\section{Literature Review}

The literature reports that the first mention of Power-to-Gas technology appeared in Japan [11,12]. In the following years there has been a rapid increase in interest in this method of storing electricity from renewable sources [9,10,13-27].

Part of the work devoted to this topic concerns the optimal size and deployment of P2G installations. In article [28], the authors present analyses concerning the optimal selection of electrolyzers size by minimizing hydrogen production costs. Maximization of profit from energy purchase and sale with simultaneous minimization of operating costs through the use of P2G technology is considered in article [29]. For the research, a 30 node IEEE 30 test network and actual historical data were used. Optimal size and distribution of electrolyzers is analyzed in paper [30]. The objective function included both the number of units and the place of their connection. An interesting analysis can be found in study [31], where the selection of the electrolyzers was considered as an alternative to network expansion. The actual distribution network was modelled and the possible size of the electrolyzers was examined in order to achieve the same effect as in the case of network modernization (e.g., cable replacement) to eliminate overloads. The results showed that the cable is now a more cost-effective option compared to installing an electrolyzers. According to the article, a cable investment is about $30 \%$ of the electrolyzer's costs. However, the profitability of an electrolyzer installation depends to a large extent on the excess power in the network. The steady decrease of unit costs of electrolyzers should also be kept in mind [32]. Article [22] focuses on reducing the constraints on wind power generation by using storage capacity. Greater capacity means more capacity to be produced from these sources. Energy storage may, thus, be used to increase the capacity installed in wind farms where this is economically justified, i.e., at a lower cost than the generation curtailment.

Some articles only discuss aspects of storing surplus energy from renewable sources and feeding it into the grid under conditions of higher demand [22,33-35]. This is also possible using P2G technology, as evidenced by article [36]. Its approach is both to store surplus electricity from renewable sources and to use it later (feed-in) to flatten the load curve (peaks in demand). The problem was treated as a task of non-linear optimization with limitations. The aim of the analyses was to maximize the profit from the operation of such a system, as well as the possibility of optimal provision of voltage control services in the grid, both in high generation states and in high power demand.

The use of "flexible loads" technology for voltage control in the MV network is presented in article [37]. Particular attention is paid to the coordination of different ways of keeping the voltage within acceptable limits. Paper [38] reviews typical solutions for elimination of negative voltage effects in networks saturated with installations of renewable energy sources. The way of their assessment is presented and what should be followed, when choosing an appropriate method.

The subject of power losses appears in article [35], where it is proposed to create an optimal charging/discharging schedule for minimizing power losses. The authors claim that the proposed method can be used to analyze other problems such as voltage control, flattening of the load curve and improving reliability.

Similar considerations are also being carried out for low-voltage networks, where an increasing number of micro-installations are being connected. An example is work [39], which presents the possibility of implementing P2G systems to absorb surplus electricity from photovoltaic installations. The example of the southern region of Germany, which has a large capacity to generate power from such sources, has been analyzed by forecasting load and generation profiles for the period 2015-2025. 
In the context of the above review, the present article is characterized by a comprehensive approach to the regulation process, since as many as three types of decision-making variables are used, and at the same time, the technological process of hydrogen production is considered. The participation of electrolyzers in the process of regulating voltage and maintaining its quality may be treated as a commercial ancillary service for the benefit of the power system.

The authors are aware that the performance of OPF (Optimal Power Flow) calculations in practice requires a network computing model in real mode (in each calculation cycle). This is achieved using a state estimation process that is enabled by the voltage and power measurements. The subject of estimation and the impact of measurement accuracy on the results of power flows has been the subject of research, among others, in the works [40-42]. It seems that the implementation of the proposed voltage control system is possible due to the small size of the computational task, and thus-the short time of obtaining the calculation results, enabling the process of optimal voltage regulation in real time. Due to the fact that the state estimation was not the main subject of this article, the authors decided to discuss this problem further in subsequent publications.

\section{Voltage Control in the MV Network with the Participation of Electrolyzers as an Optimization Task}

\subsection{Formulation of the Objective Function and Its Constrains}

The purpose of voltage control presented in this article is not only to maintain the voltages in network nodes within the scope required by the regulations [43,44], i.e., between $0.9 \cdot U_{\mathrm{nMV}}$ and 1.1. $U_{\mathrm{nMV}}$, but also to equalize the voltage profile of the entire network. Supplying the receivers with a voltage of a different value than the nominal one affects the deterioration of their efficiency, durability and reliability. Therefore, one of the most important objectives of the control carried out in the distribution network is to keep the voltage as close as possible to the nominal value in all its nodes. It is not possible to precisely achieve this goal in every node of the network, due to changing loads and generation of active power and changes of voltage values on the HV side. The optimization process allows for its "best" practical implementation.

Therefore, the traditional standard of MV network operation consists in maintaining day and night value (set) of voltage on MV buses in a HV/MV station by changing the transformer taps under load. Such a state (in Polish conditions, two voltage settings are applied-day and night-within the range from $15.5 \mathrm{kV}$ to $16.3 \mathrm{kV}$ ) does not, of course, guarantee optimal conditions of network operation or reaching the values of voltages close to the nominal one in all network nodes. The HV/MV power supply station generally consists of two transformers with 10-25 MVA each. Several to a dozen of MV lines are connected to the MV switchgear (usually $15 \mathrm{kV}$ in Poland). Each line supplies at least a dozen or so MV/LV transformer stations-Figure 1. The total number of transformer stations supplied from one HV / MV power supply station is usually several dozen, often more than a hundred. Since, in most cases, the transformers in the station work on separate sections of the MV switchgear, the evaluation of the voltage quality is formulated in relation to the area supplied from one transformer. In such a network area, there appear objects of dispersed generation, whose influence on voltage conditions is significant. Sometimes these objects are planned to be connected in nodes of one MV line. Such an example is analyzed in the present article.

The parameter that characterizes the quality of the voltage supply to costumers in static conditions is the voltage deviation. As has already been stated, the most desirable value, from the point of view of operation and lifetime of the costumers, is the voltage close to the nominal one. Maintaining the voltage value within the acceptable range $\left( \pm 10 \% U_{n}\right)$ does not guarantee optimal operating conditions for the costumers. Due to the changing load of the network and voltage drops occurring, day or night setting on MV buses in $\mathrm{HV} / \mathrm{MV}$ station $\left(U_{\mathrm{MVs}}=\right.$ const) also does not guarantee the operation of costumers in optimal voltage conditions. Therefore, various voltage quality indicators 
are introduced, allowing for global assessment of network voltage conditions and their optimization [2,4,6,45-47].

The analyses carried out assume that the expected voltage value for all nodes of the MV network is $15.75 \mathrm{kV}\left(1.05 \cdot U_{\mathrm{nMV}}\right)$ and a quality indicator of the regulation process has been proposed,

$$
F(x, y, z)=\sum_{i=1}^{N}\left(\frac{U_{i}-U_{\mathrm{o}}}{U_{\mathrm{n}}}\right)^{2}
$$

where: $U_{\mathrm{o}}$-expected voltage value in all network nodes equal to $1.05 \cdot U_{\mathrm{nMV}}$.

It was assumed that each state of the network depends on the value of the elements of three vectors $-x, y, z$. Their physical sense is presented below:

$\rightarrow \quad x=\left[\vartheta, \mathrm{Q}_{\mathrm{G} 1}, \ldots \mathrm{Q}_{\mathrm{G} k}, \ldots Q_{\mathrm{G} p}, P_{\mathrm{GE} 1} \ldots P_{\mathrm{GE} p}\right]$ 一a vector of decision (control) variables, which is formed by: The HV/MV transformer ratio ( $\vartheta$-discrete variable), reactive power $p$ of sources connected to the MV grid and active power $p$ of electrolyzers,

$\rightarrow y=\left[U_{\mathrm{HV}}, P_{\mathrm{L} 1}, \ldots P_{\mathrm{L} m}, Q_{\mathrm{L} 1}, \ldots Q_{\mathrm{L} m}, P_{\mathrm{G} 1}, \ldots P_{\mathrm{G} p}\right]$-a vector of independent variables formed by: HV network setpoint voltage, active and reactive power received in $m$ nodes and power generated in $p$ sources, not subject to change during optimization process,

$\rightarrow \quad z=\left[U_{1}, \ldots U_{j}, \delta_{1}, \ldots \delta_{j}\right]$ - the dependent variable vector (also known as the state variable vector) formed by the nodal voltages and their arguments (total number of network nodes $j=p+m$ ).

Considering the objective function described above, together with the vectors $x, y, z$, the optimization task under consideration can be formulated as follows:

- the vector of decision variables $x$ providing the minimum function described by the relation (1) is sought:

$$
F(x, y, z) \rightarrow \min
$$

- the vector of inequality limits $\mathbf{h}$, ensuring that the value of the vector elements of dependent variables and the vector of decision variables is maintained within the limits set by technical requirements:

$$
\mathbf{h}(x, y, z) \geq 0 .
$$

Inequality constrains were defined as follows:

- $\quad$ minimum and maximum values for transformer ratio $(\vartheta)$. The transformer adopted for the calculation has 19 operating positions of the on-load tap changer, within \pm 9 (including the tap in the neutral position).

- $\quad$ minimum and maximum reactive power values of each renewable source $\left(Q_{G K}\right)$. It is assumed that each RES source has the ability to generate/absorb maximum reactive power equal to $0,4, P_{n G}$,

- $\quad$ minimum and maximum voltage values for all network nodes $\left(U_{i}\right)$. The voltage was maintained in the range from $0.9 U_{\mathrm{nMV}}$ to $1.1 U_{\mathrm{nMV}}$, which translates into voltage values from $13.5 \mathrm{kV}$ to $16.5 \mathrm{kV}$,

- the permissible current-carrying capacity values for sections of power lines $\left(I_{l \max }\right)$. The value assumed in the calculations was $I_{l \max }=290 \mathrm{~A}$,

- the permissible load of the transformer $\left(S_{n T}\right)$. The calculations assume the transformer power $S_{n T}=10$ MVA.

The determination of the state vector $z$, for the known values of vectors $x$ and $y$, is one of the basic computational problems included in the computer analysis of power systems. This problem, known as load flow analysis (LF), is described in textbooks, such as $[48,49]$. 
Many algorithms and computer programs have been developed to solve this problem. Generally speaking, the components of the unknown vector $z$ (state variables) are determined from a system of nonlinear equations of the form:

$$
0=f_{\mathrm{LF}}(x, y, z)
$$

Each equation is formulated for a node of the analyzed network, so that the power balance for this node is equal to zero.

The nature of the issue under consideration is such that the determination of the elements of the state vector takes place through an iterative process. The decision variable vector consists of a discrete variable (transformer tap changer setting) and continuous variables (reactive power of sources, active power of electrolyzers). The use of the classical optimization method to solve the problem under consideration would be difficult to implement, so one of the heuristic methods was chosen, which is described in point 3.2.

\subsection{Description of the Optimisation Algorithm}

The task under consideration is known as OPF (Optimal Power Flow). In view of the difficulties with quick solution of OPF type tasks using methods similar to the classical ones [50-53], which have been found in the research and confirmed in many publications, attention was focused on the possibility of using an alternative method of solving problems of non-linear optimization, which is based on heuristics. Heuristic methods allow to solve various types of tasks that cannot be solved by classical methods or the application of these methods is too time-consuming and complicated in the software sense. Compared to classical methods, heuristic methods are characterized by the fact that they do not require knowledge of the derivative form of the objective function, they are resistant to discontinuities of this function and to "getting stuck" of the calculation process in the local minimum. In case of a divergent iterative process, the heuristics work in such a way that the flow data can be reloaded at any time during the calculation process. The best solution recently found is not lost, as this vector is remembered at every stage of the calculation. The classic method would then have to be interrupted and the calculation restarted. Moreover, heuristic methods can start from an unacceptable point (a penalty for exceeding the limits is then added).

Heuristic methods are currently used more and more often (not only in the power industry), as modern gradient-less methods to solve non-linear optimization tasks with limitations. They are constantly being developed, as evidenced by new publications [54-58]. Sources of inspiration in the development of new methods may be different. Solving optimization tasks, belonging to different fields of science and technology, does not require fundamental changes in algorithms. In particular, every objective function can be optimized without the need to check its continuity or differentiation. Due to the random nature of heuristic methods, there is a high probability of finding a global optimum.

The optimization method used in the research is called the Algorithm of the Innovative Gunner (AIG) [59]. It differs from other methods of generating new values of decision variables. Generally speaking, in most heuristic methods, the iterative process of determining new values of the vector components $x$ is similar, because most often in the next iterative step an action [53,60-63], is performed, which can be described as an additive modification,

$$
x_{l}^{(i+1)}=x_{l}^{(i)}+\Delta x_{l}^{(i)}
$$

and the correction of the $l$-th component of the vector, in the $i$-th iterative step, is determined as a function value,

$$
\Delta x_{l}^{(i)}=f_{l}(\xi)
$$

whereby the $f_{l}$ function and the $\xi$ vector are characteristic of the heuristic method used. 
In the case of the AIG algorithm, relation (5) is replaced by an expression:

$$
x_{l}^{(i+1)}=x_{l}^{(i)} \cdot g_{l 1}\left(\xi_{1}\right) \cdot g_{l 2}\left(\xi_{2}\right) .
$$

Therefore, the difference is that in the next steps the components of vector $x$ change by multiplying (no by adding). The $g_{l 1}\left(\xi_{1}\right), g_{l 2}\left(\xi_{2}\right)$ functions, providing that $\xi_{1}=\alpha, \xi_{2}=\beta$, are assumed accordingly:

$$
\begin{array}{ll}
g(\alpha)=\csc (\alpha)=(\cos (\alpha))^{-1} & \text { for } \alpha>0 \\
g(\alpha)=\cos (\alpha) & \text { for } \alpha<0 \\
g(\beta)=\csc (\beta)=(\cos (\beta))^{-1} & \text { for } \beta>0 \\
g(\beta)=\cos (\beta) & \text { for } \beta<0
\end{array}
$$

where $\alpha$ and $\beta$ are the "correction angles", drawn from the $\left(-\alpha_{\max }, \alpha_{\max }\right)$ and $\left(-\beta_{\max }\right.$, $\beta_{\max }$ ) range using a normal (or uniform) probability distribution.

The block diagram of the AIG algorithm is shown in Figure 2 [59].

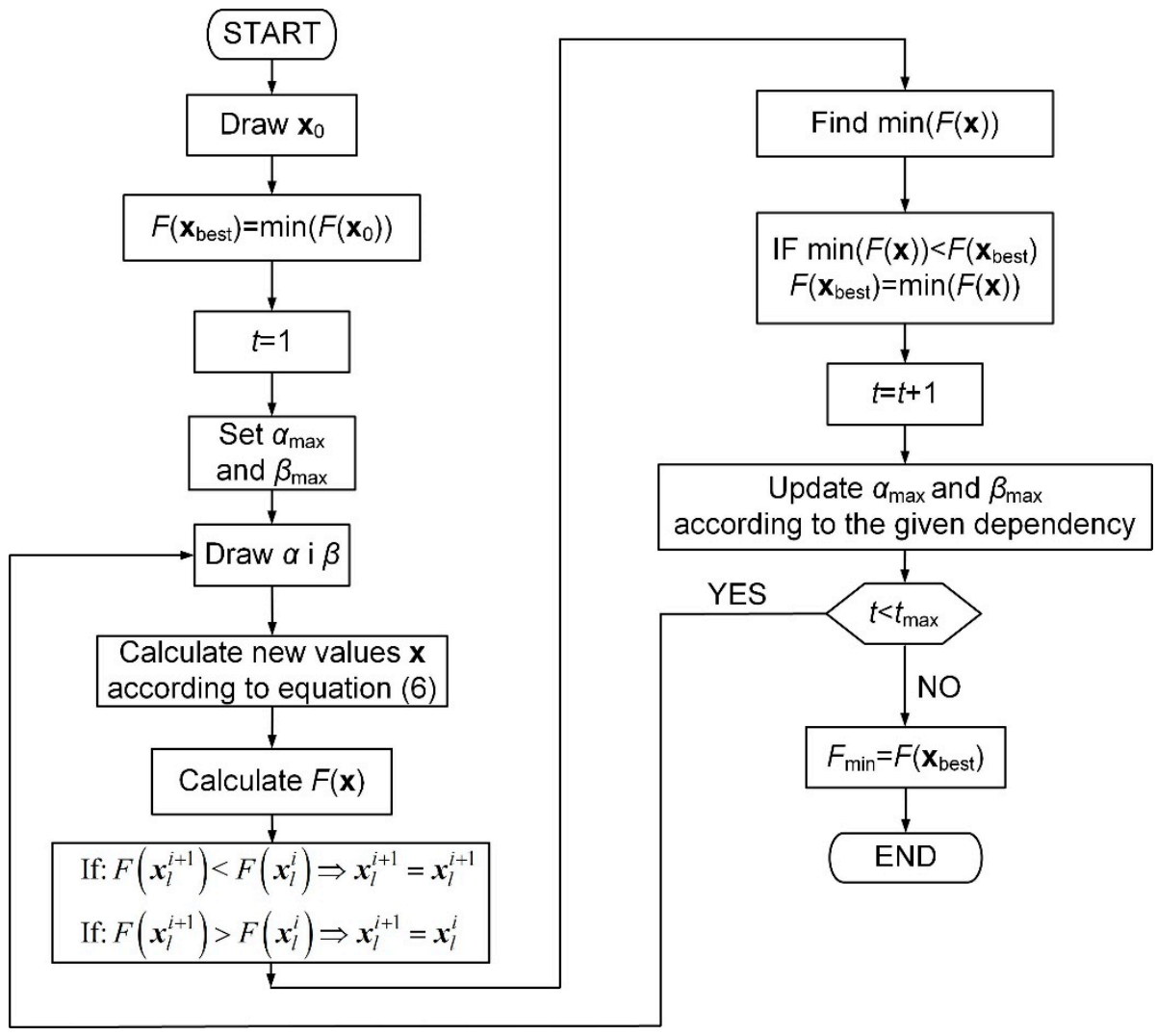

Figure 2. AIG algorithm block diagram ( $t$ is the next iteration).

Given that in the AIG algorithm we are dealing with a population of new solutions, it is characterized by a large exploration of the search space. The subsequent solutions differ (sometimes quite significantly) from the previous ones, which allows to avoid local optimum. Its innovativeness results from a specific and original way of determining subsequent solutions in the iterative process, which distinguishes it from other heuristic methods. The presentation of the AIG algorithm and its properties was made by the authors in [59], and its effectiveness was widely studied in [53].

In order to solve problems in the field of power engineering an appropriate calculation workshop is necessary. The PowerWorld Simulator program, version 20, was used for calculations. This program is mainly used to calculate power flows in the power system. The software includes the SimAuto add-on, which enables connecting to external applica- 
tions (e.g., Matlab, Excel, Delphi). Notably, SimAuto acts as an interchangeable calculation engine that can be used in various applications. This article uses the Matlab environment, where a script is written that connects to SimAuto and calls the AIG algorithm. The course of the calculation is shown in Figure 3. The application is universal and can be used to solve various optimization issues. After running the main script in Matlab environment, there is a remote connection with the load flow program (PowerWorld Simulator) via SimAuto add-on. The change of parameters of the grid elements or the downloading of calculation results is carried out by means of appropriate commands, suitable for the respective development environment. When the AIG algorithm is started, optimization calculations follow, after which the results are saved in a file.

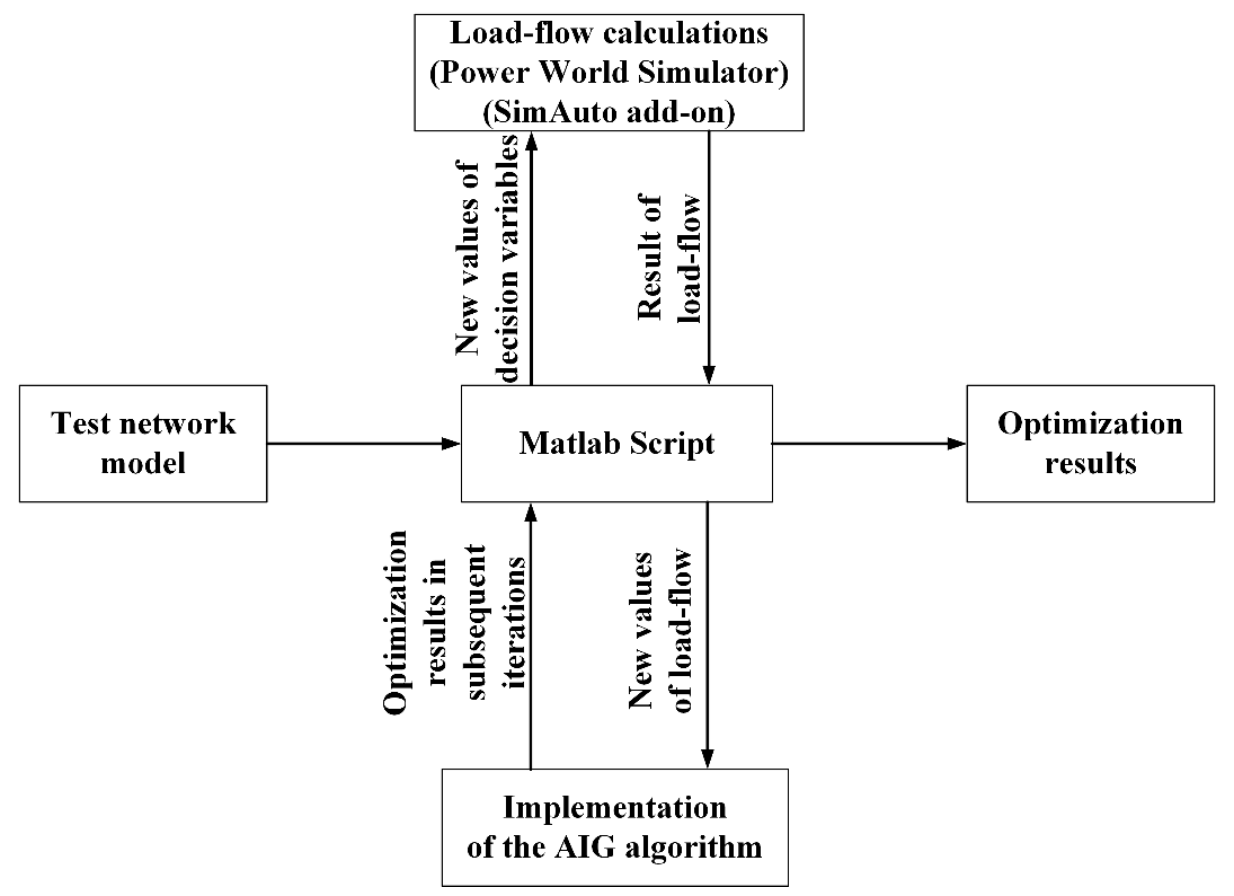

Figure 3. General scheme of the organization of the computational process used in solving the optimization task.

\section{Description of the Test Network}

For the analyses, the IEEE 37 [64], network was used, whose parameters were adjusted to the MV network, typical of Polish conditions. It was assumed that the total length of individual sections of the $15 \mathrm{kV}$ overhead line with branches is $40 \mathrm{~km}$. The line is made with ACSR $70 \mathrm{~mm}^{2}$ conductors-Figure 4 . There is a transformer with a power of $10 \mathrm{MVA}$ and a ratio $\vartheta=115 / 16.5 \mathrm{kV} / \mathrm{kV} \pm 10 \%$ (taps every $1.11 \%$ ). There are five sources connected to the lines with power ratings of $P_{\mathrm{nG} 1}=0.5 \mathrm{MW}, P_{\mathrm{nG} 2}=0.5 \mathrm{MW}, P_{\mathrm{nG} 3}=2.0 \mathrm{MW}, P_{\mathrm{nG} 4}=1.0 \mathrm{MW}$, and $P_{\mathrm{nG} 5}=2.0 \mathrm{MW}$, respectively. Each source has the capacity to generate reactive power in the range $(-0.4,0.4) P_{\mathrm{nG}}$ and this power is remotely controlled in the system shown in Figure 1.

The network load includes MV/LV transformer stations connected in almost all nodes (the total number of nodes is $m=37$ ), with the exception of nodes $0,10,16,25,29$ and 34 (Figure 4). In the mentioned nodes (except node 0 ), electrolyzer installations are connected (marked in green). For electrolyzer installations, the rated power was not determined so that their power demand would result from control needs. The use of ALE-type electrolyzer properties and modular construction of the installation allow for power changes in the range from zero to the nominal value (unspecified in these tests), in the remote control system shown in Figure 1. The maximum load of individual nodes is shown in Figure 5d. 


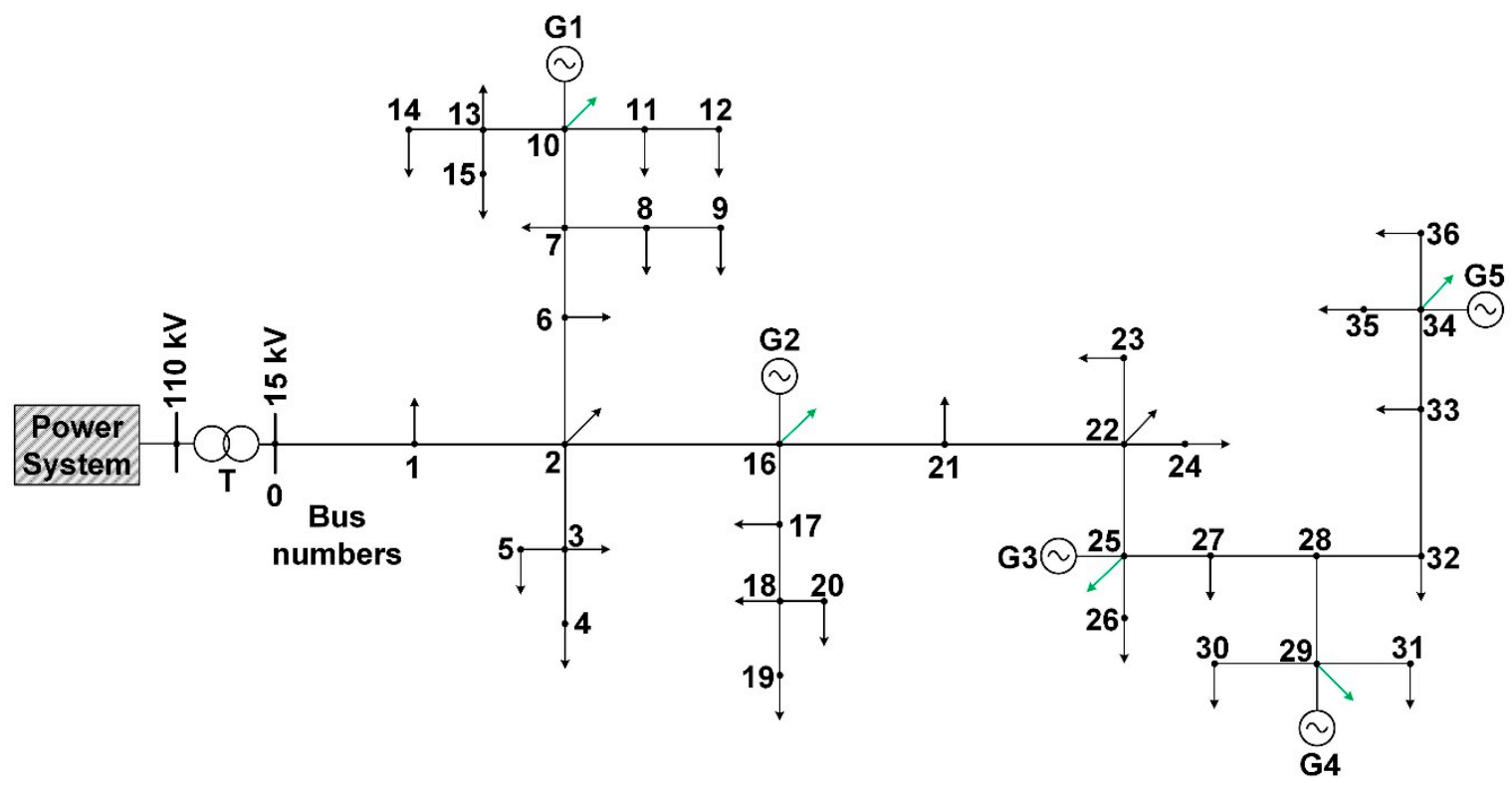

Figure 4. Test network diagram.

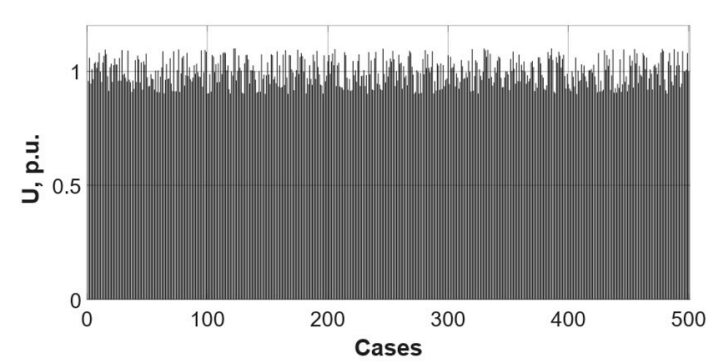

(a)

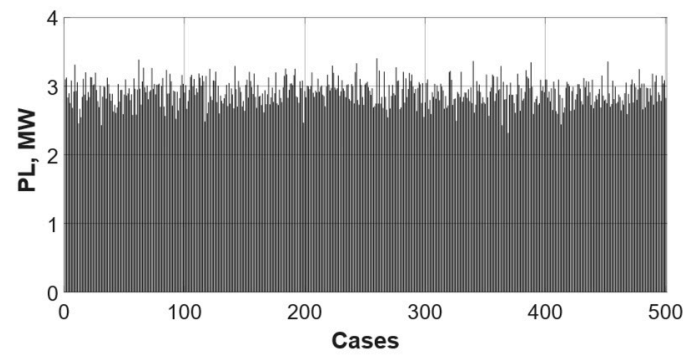

(c)

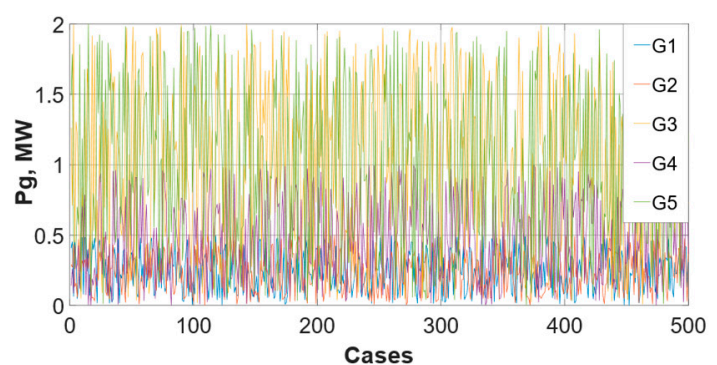

(b)

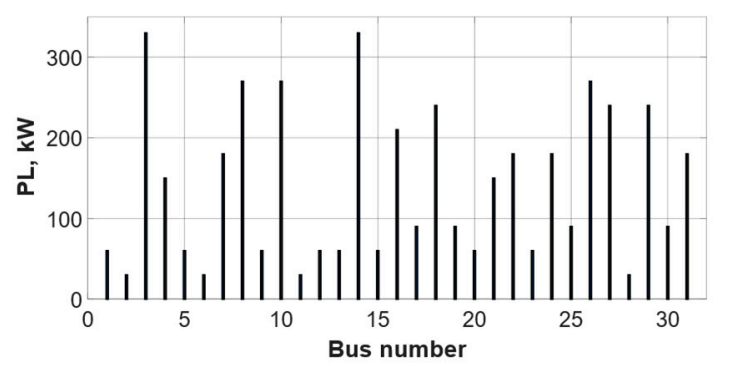

(d)

Figure 5. Drawn variable values for subsequent calculation cases (a) UHV values, (b) drawn active power of the generators, (c) total power received in the receiving nodes resulting from drawing, (d) maximum loads of individual nodes.

As mentioned above, the original IEEE 37 [64] network was adjusted to the parameters of the MV network, which is a standard solution in the Polish power system.

A single solution of the voltage control optimisation task would not allow for a reliable analysis of the problem under consideration due to the variability of operating conditions of the test network examined (changes of HV voltage, load power and power generated in distributed sources). Therefore, it was decided to repeat the calculations many times (five hundred calculation cycles) for different operating conditions of the MV network. Being aware of the fact that individual variables may be subject to different probability distributions, it was decided that their values should be randomised using a random number generator with rectangular distribution. In this way, the authors wanted to take into account all possible cases of mutual numerical relations between the quantities influencing the network. This will happen when every value of independent variables is 
equally probable, with no correlation between their values. Voltage values on the $110 \mathrm{kV}$ $\left(U_{\mathrm{HV}}\right)$ side were drawn from $0.9 \cdot U_{\mathrm{nHV}}$ to $1.1 \cdot U_{\mathrm{nHV}}$, which corresponds to a voltage between $99 \mathrm{kV}$ and $121 \mathrm{kV}$. The load in MV network nodes was drawn in the range from $30 \%$ to $100 \%$ of maximum load, shown in Figure 5d. The power generated in RES was drawn in the range from 0 to $P_{\mathrm{nGK}}$.

Figure $5 \mathrm{a}$ presents the values of $U_{\mathrm{HV}}$ voltage for a cycle of five hundred draws, Figure $5 \mathrm{~b}$ presents the drawn values of the power generated in RES, and Figure 5c presents the total value of the power received in the receiving nodes resulting from the draws. The maximum values of the power absorbed at individual MV/LV stations range from $30 \mathrm{~kW}$ to $330 \mathrm{~kW}$. The total load on the grid (without electrolyzers) ranges from $2.32 \mathrm{MW}$ to $3.41 \mathrm{MW}$. It is clear that the maximum value of the power consumed in the grid is lower than the maximum power that can be generated from renewable sources. Therefore, periods can be expected when power flows towards the HV/MV transformer station. Such a state of operation is associated with the effect of voltage increase and the possibility of reaching a value higher than allowed, in MV nodes. Then electrolyzer installations should fulfil their task. Excess power from renewable sources should be absorbed by them and in this way the voltage problem will be eliminated. The cooperation of electrolyzers with RES' will allow not only to maintain the values of voltage within the acceptable range, but also to achieve their optimal value from the point of view of the quality indicator, defined by Formula (1). As indicated by simulation analyses made in order to optimize (concentrate) the values of voltages in all network nodes near the value of $1.05 U_{\mathrm{nMV}}$, the variation of reactive power of the sources and active electrolyzers, resulting from optimal voltage regulation, is a much more complex process than the qualitative description presented above.

\section{Results of Voltage Optimization in MV Networks}

Optimization calculations were performed using a single-criteria target function, described by Equation (1), defining the voltage quality indicator. The optimization process is aimed not only at keeping the voltage values in MV network nodes within acceptable limits, but also at equalising the voltage profile, i.e., concentrating the voltage values in all nodes around 1.05. $U_{\mathrm{nMV}}$. The calculations were performed for five hundred network operating states by random change of voltage on the HV side of the transformer, drawing the value of active power generated in each of the five RESs and load in all MV/LV transformer stations. The calculations considered the state with traditional control carried out by OLTC (voltage on MV buses maintained at the level of $1.05 U_{\mathrm{nMV}}$ ), taking into account simultaneous control of RES reactive power and active power of electrolyzer installations (full optimization) and without the participation of these installations (OLTC only and reactive power-partial optimization).

Figure $6 \mathrm{a}-\mathrm{c}$ show the values of voltages corresponding to the above-mentioned control methods (before and after optimization-full, and partial optimization, respectively), for all, five hundred random network operation states. Due to the generation in RESs, which in some cases exceeds the total load of the grid, the values of the voltages in the state before optimization (Figure 6a), in some states exceed the permissible range. Even a qualitative assessment of the "ribbon" that forms the lines corresponding to the individual calculation cycles clearly indicates a reduction in its area and a change in its shape, the greatest for full optimization, a little less for partial optimization. The maximum value of the voltage quality indicator before optimization was 0.055691, and its average value was 0.009135, while after optimization (in a system with electrolyzers-Figure 6b), in each state of the network, the control system "tries" to keep the voltage close to the expected value, i.e., $1.05 U_{\mathrm{nMV}}$. The voltage quality indicator in each case improves several times in relation to the state before optimization-Figure $7 \mathrm{a}$. The maximum value of the voltage quality indicator after full optimization was 0.001708 and its average value was 0.000417 . Thus, after optimization, the average value of the voltage quality indicator decreased more than twenty-one times (for the vertical axis calibrated as in Figure 7a, the values of the voltage quality indicator are very small and not very visible-brown colour). For comparison, 
similar calculations were made for partial optimization status (OLTC only and reactive power of RES). The maximum value of the voltage quality indicator was 0.004984 and its average value was 0.00065 . Thus, after the optimisation, the average value of the voltage quality indicator decreased fourteen times. Figure $7 \mathrm{c}$ compares the effects of full and partial optimisation. It can be clearly seen that the lack of installation of electrolyzers affects the deterioration of the voltage profile of MV network, thus, it should be considered that the active power of the installation ALE contributes significantly to the quality of the voltage control process. The average value of power losses in the analysed network also decreased-from $42.1 \mathrm{~kW}$ to $29.25 \mathrm{~kW}$ (in an optimised system with electrolyzers). This is important because the value of power losses was not part of the minimised objective function. Although globally the power losses decreased, states could be observed where they were slightly higher than before optimization-Figure $7 \mathrm{~b}$. For comparison, analogous calculations were also made for conditions without electrolyzers. The average power losses increased to $76 \mathrm{~kW}$. In some cases, they were larger than the pre-optimization conditionFigure $7 \mathrm{~d}$. Thus, power control of loads has a significant impact on the reduction of power losses in the grid.

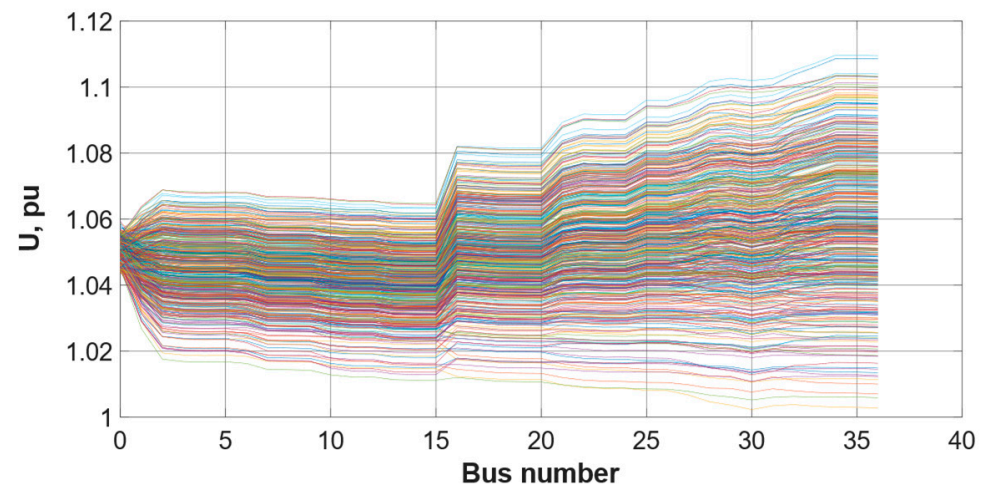

(a)

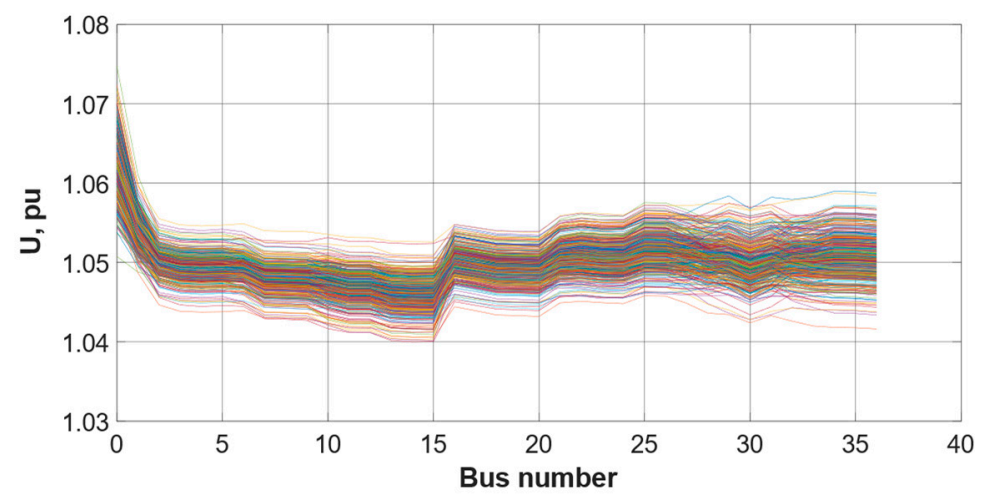

(b)

Figure 6. Cont. 


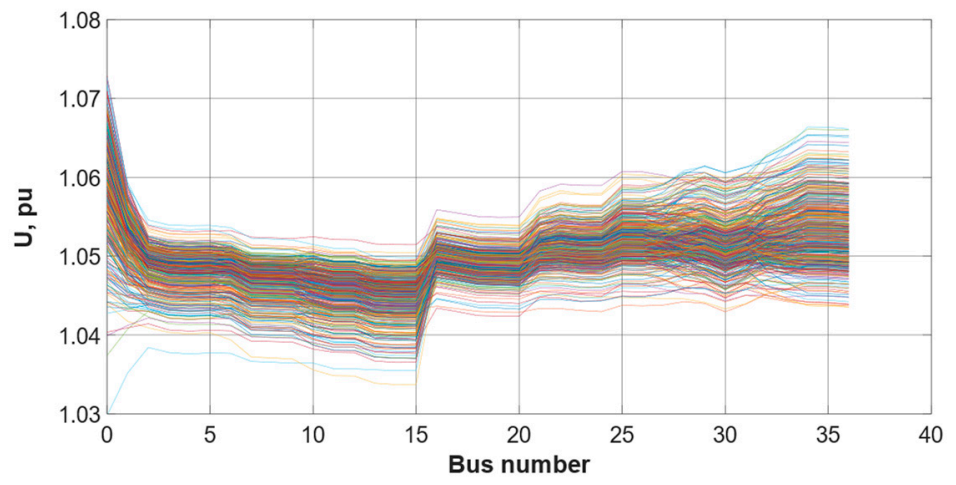

(c)

Figure 6. Voltage values at all MV network nodes, control according to $1.05 \cdot U_{\mathrm{nMV}}, 500$ calculation cases, (a) before optimization, (b) after optimization (including electrolyzer installations), (c) after optimization (excluding electrolyzer installations).

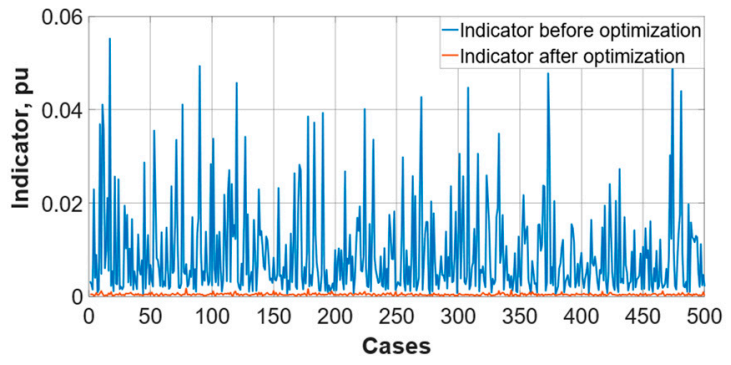

(a)

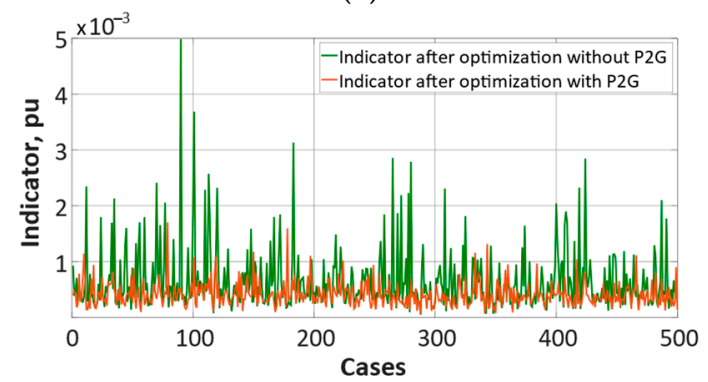

(c)

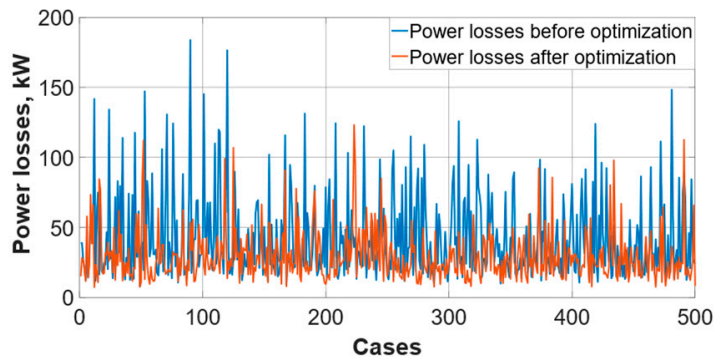

(b)

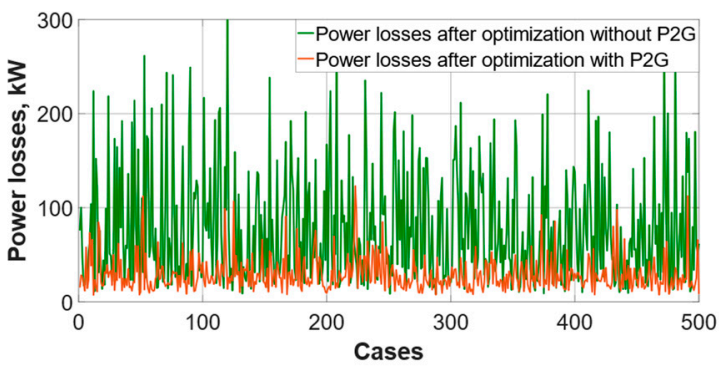

(d)

Figure 7. Comparison of values of: (a) voltage indicator (without optimisation and full optimization), (b) power loss (without optimization and full optimization), (c) voltage indicator (full optimization and partial optimization), (d) power losses (full optimization and partial optimization) for 500 calculation cases.

Figure 8 shows the changes of the HV/MV transformer taps (Figure 8a-full optimization, Figure $8 \mathrm{c}-$ partial optimization) and the reactive power of the generators (Figure $8 \mathrm{~b}$-full optimization, Figure $8 \mathrm{~d}$-partial optimization). For a significant number of cases the optimal position of the on-load tap-changer is equal to -9 . The generators with the highest power (G3 and G5) either generate reactive power more often (G3) or, as in the case of (G5), consume reactive power more often, which can be justified by their position in the network (G5 lies far from the HV/MV station). Both of these observations indicate an aspiration to maintain the voltage in the network at $1.05 U_{\mathrm{n}}$, despite significant power consumption (active and reactive). In the state without the installation of electrolyzers (partial optimization), maximum reactive power is more often consumed, which proves the need to reduce the voltage values. This case is also a proof that the power generated in RE sources (exceeding the power consumed) flows in the direction of HV/MV stations, which causes an increase in the voltage value in the network nodes. In a system with full optimization the changes in reactive power are smaller, which (as noted above) reduces power losses. 


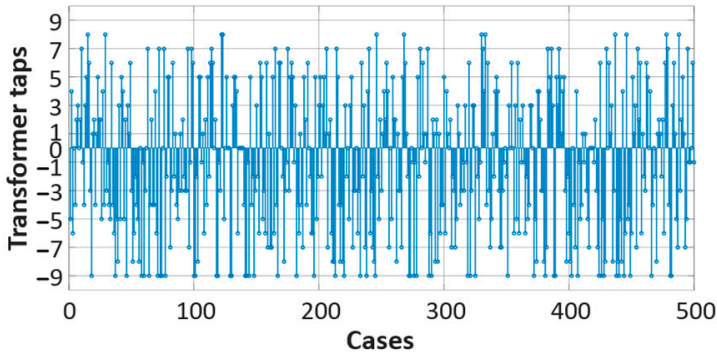

(a)

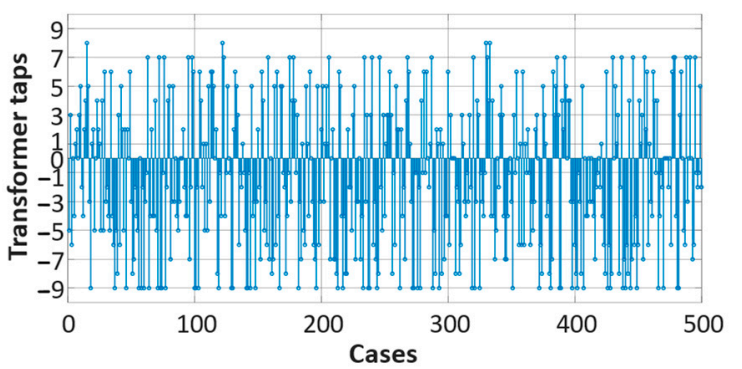

(c)

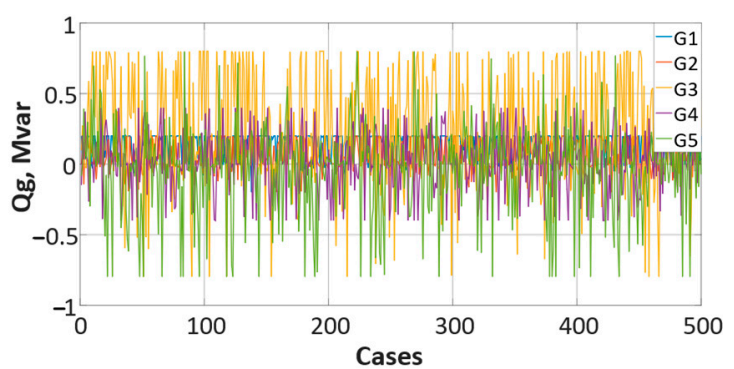

(b)

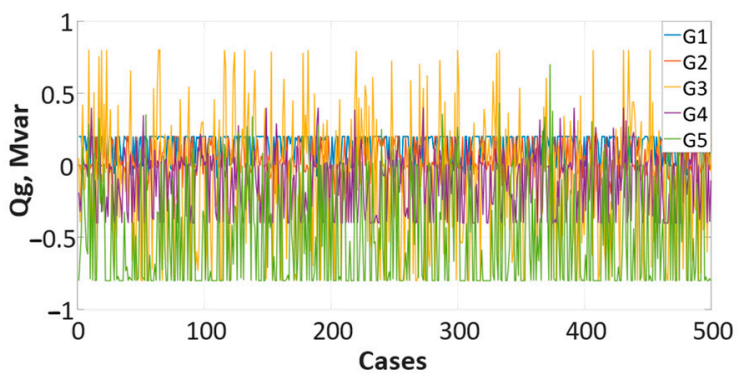

(d)

Figure 8. Variability of (a) HV/MV transformer taps-including full optimization, (b) reactive power of generatorsincluding full optimization, (c) HV/MV transformer taps-including partial optimization, (d) reactive power of generatorsincluding partial optimization.

Figure 9a-e show a comparison of active power consumed by electrolyzer installations and generated in sources. As expected, they are smaller than the generation in the sources. It is also characteristic that the closer the source is connected to the HV/MV station, the lower the power consumed by electrolyzers. It is caused by the fact that the closer to the station, the smaller the voltage impact of the sources in the grid. Therefore, the power consumed by electrolyzer installations is also relatively lower, provided that this power consumption is fully subordinated to control objectives. In this way, it is possible to control voltage problems in the radial network, in which the sources connected in the depths have the greatest influence on the effect of voltage increase. In the classic approach, the active power of the sources should be limited, which would result in financial losses as a result of lost benefits from unsold electricity.

Figure 10 shows the total power generated in dispersed sources and the total power consumed by electrolyzer installations. As can be seen, the relationship between these capacities is difficult to explain intuitively and qualitatively. They result from the optimization process, which even for a relatively small size of the task generates values which can only be verified by checking the value of the target function, i.e., indicator (1).

This chapter presents the results of calculations performed mainly with the AIG algorithm. Additionally, for comparison and verification, Figure 11 shows the course of the optimization process, also according to another heuristic algorithms-cuckoo search (CS-[65]) and moth-flame optimization (MFO-[63]). Tables 1-3 show the optimization results for one random state, also for the AIG, CS and MFO algorithms.

The graphs show that practically fifty iterations are sufficient to find the optimal solution (also for the use of CS and MFO algorithms), so the optimization process runs effectively. The test network used for the calculation, which includes both variable voltage values on the HV side, load with changing values and random generation in renewable sources, justifies the advisability of using heuristic optimization methods. In the future, connecting an increasing number of RES with variable generation to the medium and low voltage grid, will require the use of more and more advanced optimization algorithms authorising the use of the term smart grid for these networks. 


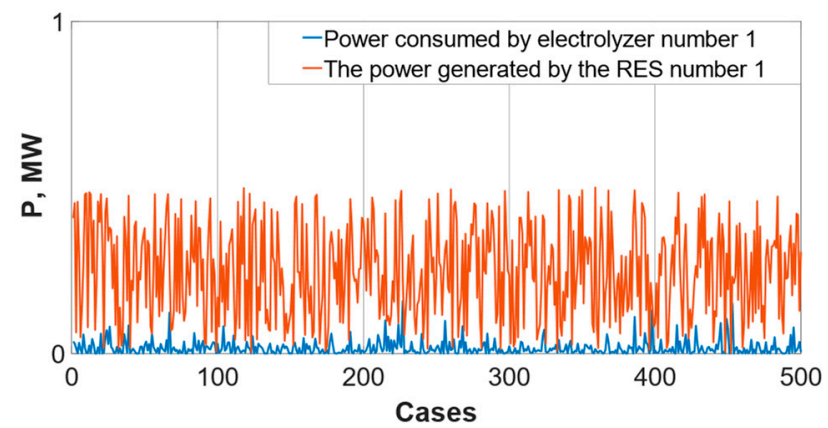

(a)

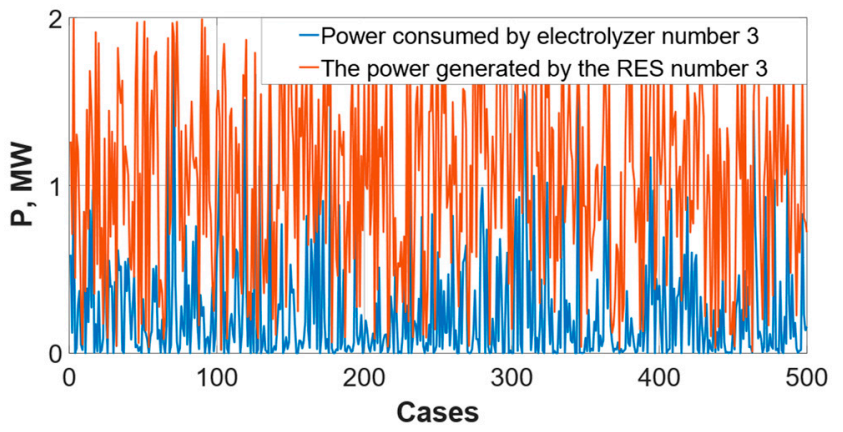

(c)

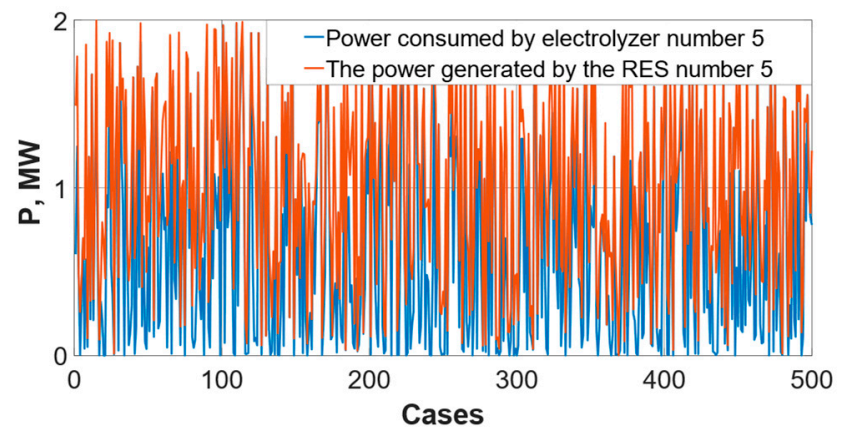

(e)

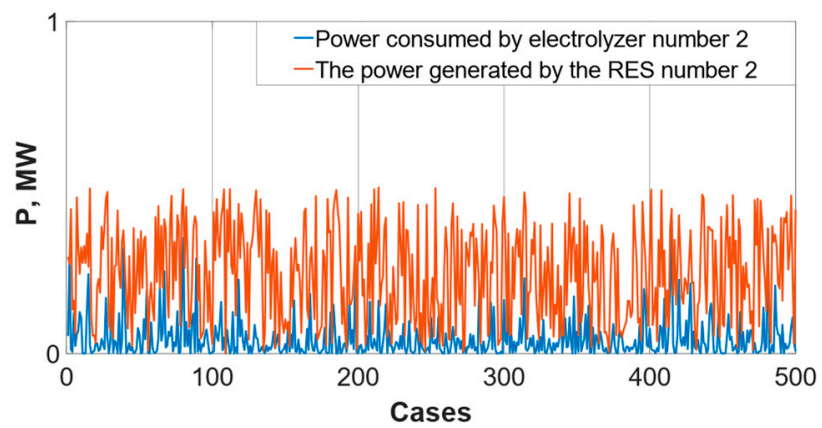

(b)

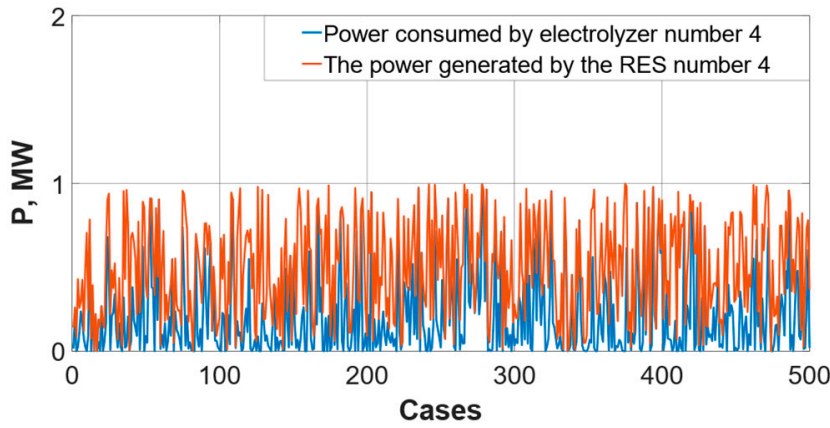

(d)

Figure 9. Comparison of power consumed by electrolyzers and power generated (a) in installation and source No 1, (b) in installation and source No 2, (c) in installation and source No 3, (d) in installation and source No 4, (e) in installation and source No 5 (full optimization).

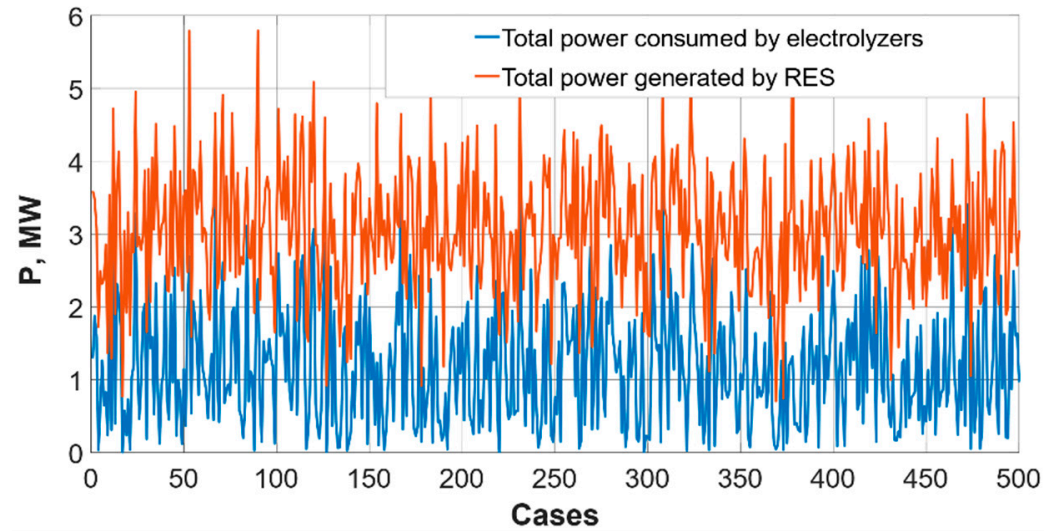

(a)

Figure 10. Cont. 


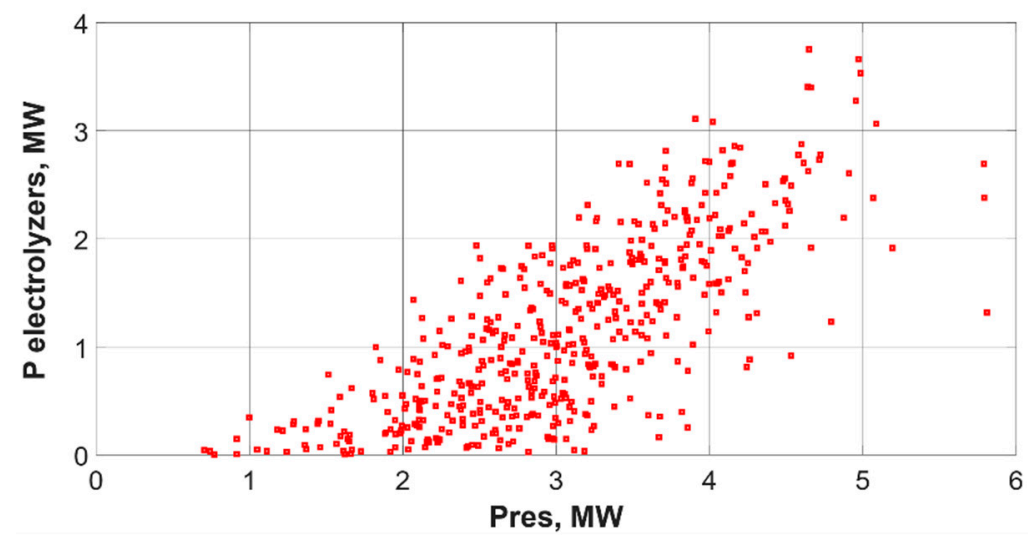

(b)

Figure 10. Power generated in RESs and power consumed by electrolyzers-500 calculation cases (a) total values, (b) dependence of total power consumed by electrolyzers on power generated (full optimization).

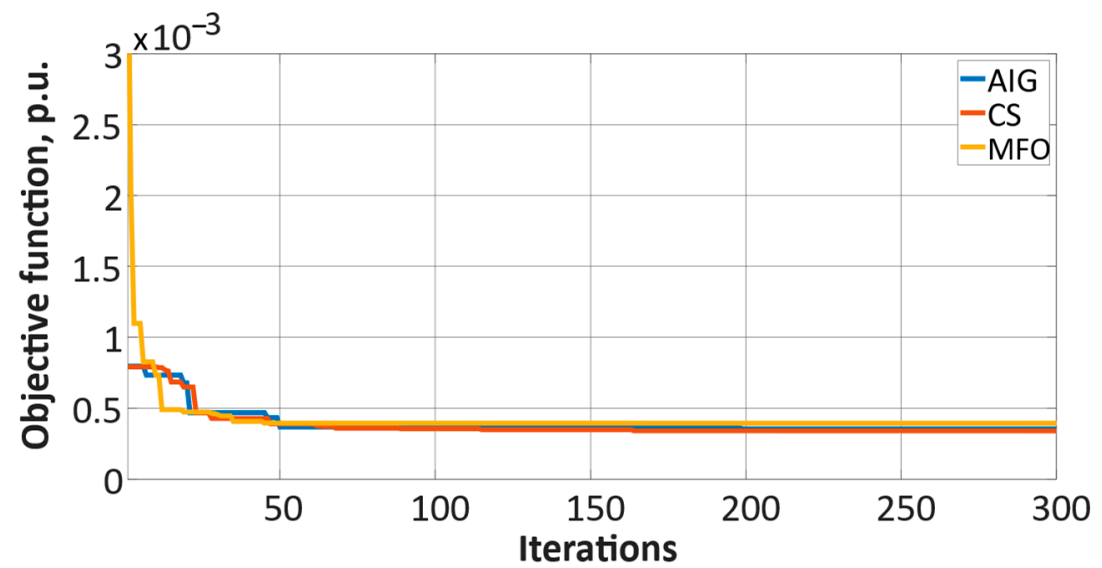

Figure 11. Voltage quality indicator changes (objective function) in subsequent iterations, for one of the calculation cases, for AIG, CS and MFO heuristic algorithms used.

Tables 1-3 show the calculation results for three optimization algorithms (AIG, CS and MFO), for one calculation cycle, randomly selected from among five hundred tested cases. The voltage value drawn on the HV side was 1.0358 p.u. It should be noted that although the obtained objective function values for all three algorithms are very similar (Table 3), the values of individual decision variables differ. This is due to the heuristic optimization property, in which probabilistic plays an important role and the final evaluation of the algorithm used results from multiple repetition of calculation cycles.

The computational tests described above were performed with the assumption that the values of the real independent variables are accurately mapped in the computational process. This is of course not the case, as transmission errors may affect the values of these variables used in the calculations. Although the issues of the state estimation and accuracy of measurement data were beyond the main scope of the presented article, an initial test of the algorithm's resistance to measurement errors of independent variables was performed. For this purpose, from among five hundred calculation cases, case number 168 was selected at random. 
Table 1. The optimal voltage values in the test network nodes for three heuristic algorithms (AIG, CS and MFO) for one of the calculation cases.

\begin{tabular}{|c|c|c|c|c|c|}
\hline \multirow{2}{*}{ Bus No. } & \multirow{2}{*}{$\operatorname{Load} P_{\mathrm{L}}, \mathrm{kW}$} & \multirow{2}{*}{$\begin{array}{c}\text { Voltage before } \\
\text { Optimization, } U \text { p.u. }\end{array}$} & \multicolumn{3}{|c|}{ Voltage after Optimization, $U$ p.u. } \\
\hline & & & AIG & CS & MFO \\
\hline 0 & 0.0 & 1.0503 & 1.0623 & 1.0607 & 1.0601 \\
\hline 1 & 37.9 & 1.0479 & 1.0547 & 1.0518 & 1.0526 \\
\hline 2 & 20.2 & 1.0468 & 1.0509 & 1.0497 & 1.0489 \\
\hline 3 & 289.9 & 1.0460 & 1.0500 & 1.0487 & 1.0480 \\
\hline 4 & 114.4 & 1.0458 & 1.0498 & 1.0483 & 1.0478 \\
\hline 5 & 52.5 & 1.0459 & 1.0499 & 1.0484 & 1.0479 \\
\hline 6 & 17.0 & 1.0455 & 1.0498 & 1.0482 & 1.0478 \\
\hline 7 & 165.1 & 1.0436 & 1.0482 & 1.0466 & 1.0462 \\
\hline 8 & 202.0 & 1.0435 & 1.0481 & 1.0466 & 1.0461 \\
\hline 9 & 28.3 & 1.0434 & 1.0478 & 1.0465 & 1.0461 \\
\hline 10 & 0.0 & 1.0421 & 1.0470 & 1.0457 & 1.0453 \\
\hline 11 & 174.8 & 1.0416 & 1.0465 & 1.0452 & 1.0448 \\
\hline 12 & 26.9 & 1.0416 & 1.0468 & 1.0452 & 1.0448 \\
\hline 13 & 29.0 & 1.0410 & 1.0463 & 1.0447 & 1.0442 \\
\hline 14 & 29.1 & 1.0409 & 1.0462 & 1.0446 & 1.0441 \\
\hline 15 & 178.4 & 1.0409 & 1.0462 & 1.0446 & 1.0441 \\
\hline 16 & 0.0 & 1.0534 & 1.0527 & 1.0500 & 1.0508 \\
\hline 17 & 37.2 & 1.0529 & 1.0522 & 1.0495 & 1.0503 \\
\hline 18 & 196.7 & 1.0524 & 1.0517 & 1.0490 & 1.0498 \\
\hline 19 & 56.3 & 1.0524 & 1.0516 & 1.0490 & 1.0498 \\
\hline 20 & 121.3 & 1.0523 & 1.0516 & 1.0489 & 1.0497 \\
\hline 21 & 65.7 & 1.0565 & 1.0531 & 1.0500 & 1.0514 \\
\hline 22 & 44.1 & 1.0576 & 1.0533 & 1.0501 & 1.0517 \\
\hline 23 & 72.2 & 1.0574 & 1.0532 & 1.0499 & 1.0515 \\
\hline 24 & 95.6 & 1.0574 & 1.0531 & 1.0499 & 1.0515 \\
\hline 25 & 0.0 & 1.0597 & 1.0540 & 1.0505 & 1.0524 \\
\hline 26 & 37.0 & 1.0596 & 1.0539 & 1.0504 & 1.0523 \\
\hline 27 & 70.3 & 1.0599 & 1.0532 & 1.0497 & 1.0515 \\
\hline 28 & 78.1 & 1.0604 & 1.0519 & 1.0484 & 1.0502 \\
\hline 29 & 0.0 & 1.0604 & 1.0513 & 1.0479 & 1.0496 \\
\hline 30 & 257.2 & 1.0588 & 1.0497 & 1.0463 & 1.0480 \\
\hline 31 & 158.2 & 1.0602 & 1.0511 & 1.0478 & 1.0495 \\
\hline 32 & 14.7 & 1.0613 & 1.0515 & 1.0478 & 1.0497 \\
\hline 33 & 234.1 & 1.0619 & 1.0512 & 1.0475 & 1.0494 \\
\hline 34 & 0.0 & 1.0629 & 1.0514 & 1.0475 & 1.0495 \\
\hline 35 & 61.3 & 1.0628 & 1.0514 & 1.0475 & 1.0495 \\
\hline 36 & 118.7 & 1.0626 & 1.0512 & 1.0473 & 1.0493 \\
\hline
\end{tabular}

Table 2. The optimal values of reactive power of sources and power consumed by electrolysis installation, for three heuristic algorithms, for one of the calculation cases (the same as in Table 1).

\begin{tabular}{cccccccc}
\hline & \multirow{2}{*}{$\begin{array}{c}\text { Power Generation, } \\
\text { Gen No. }\end{array}$} & \multicolumn{3}{c}{$\begin{array}{c}\text { Reactive Power after } \\
\text { Optimization, Mvar }\end{array}$} & \multicolumn{3}{c}{$\begin{array}{c}\text { Power Consumed by } \\
\text { Electrolyzers MW }\end{array}$} \\
\cline { 3 - 8 } & & AIG & CS & MFO & AIG & CS & MFO \\
\hline G1 & 0.041 & 0.200 & 0.200 & 0.200 & 0.003 & 0.000 & 0.000 \\
G2 & 0.465 & 0.200 & 0.200 & 0.200 & 0.000 & 0.000 & 0.008 \\
G3 & 1.551 & 0.068 & 0.194 & 0.041 & 0.406 & 0.722 & 0.285 \\
G4 & 0.487 & -0.012 & -0.128 & -0.012 & 0.314 & 0.105 & 0.239 \\
G4 & 0.872 & -0.383 & -0.333 & -0.614 & 0.206 & 0.295 & 0.000 \\
\hline
\end{tabular}


Table 3. The optimal values of tap changer position and objective function, for three heuristic algorithms, for one of the calculation cases.

\begin{tabular}{ccc}
\hline Heuristic Algorithm & Tap Changer Position & Value of the Objective Function \\
\hline AIG & 2 & 0.000378 \\
CS & 2 & 0.000375 \\
MFO & 2 & 0.000380 \\
\hline
\end{tabular}

Treating it as a reference case, fifty additional draws of all independent variables were made (voltage values in the HV node, power received in nodes and active powers of renewable sources). For each independent variable from case 168 taken as the mean value $\mu$ of the normal distribution with standard deviation equal to $\sigma=1 \%$, its measurement representation was drawn. Therefore, the independent variables taken for the optimization calculations came in the range from 0.97 to 1.03 in relation to the reference value, which can be considered a pessimistic estimation of the transmission accuracy. Figure 12 shows the values of the voltage quality index (determined by the relation (1)) for three calculation variants:

- $\quad$ without optimization, for a reference case (straight line, blue color);

- after optimization, for the reference case, assuming an exact mapping of independent variables in the calculations (straight line, green color);

- after optimization, for fifty subcases of inaccurate transmission of the values of the independent variables, with respect to the reference case.

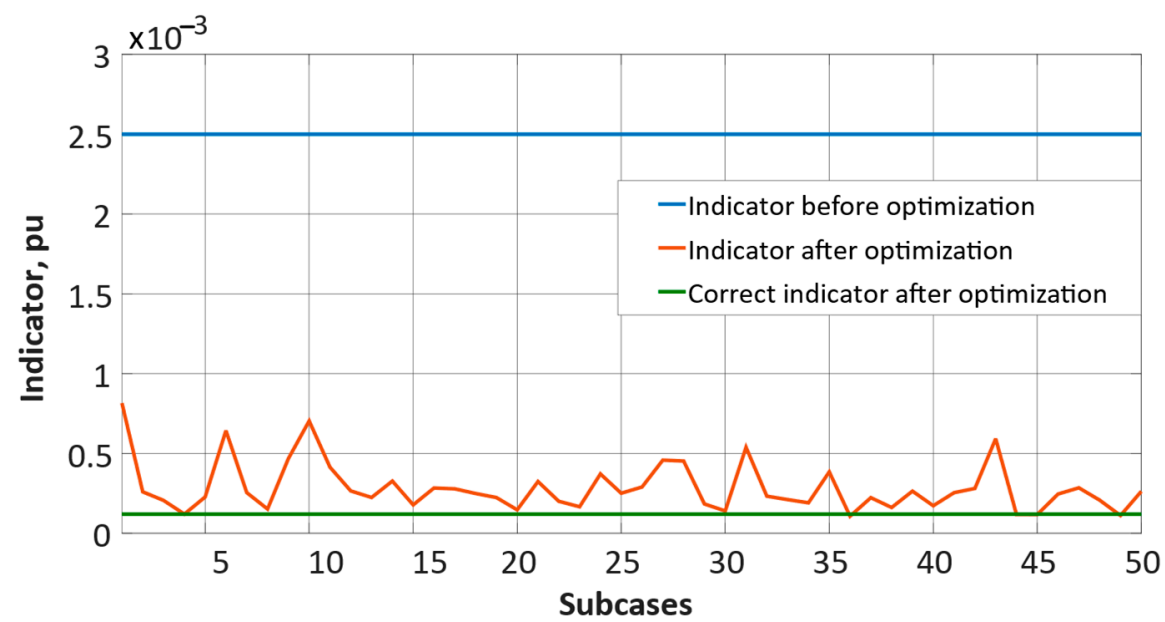

Figure 12. Illustration of the influence of mapping errors of independent variables on the result of the optimization process; case number 168 taken as reference.

On the basis of the obtained results, it can be concluded that although the mapping errors of the independent variables noticeably deteriorate the value of the voltage quality index, its improvement is still very clear compared to the case without optimization.

\section{Discussion and Conclusions}

This article draws attention to the negative voltage impact of a large number of distributed sources connected to the medium voltage grid. Their location at different points on MV lines and the possibility of maximum generation during off-peak hours may lead to voltage variations over a wide range. Sometimes the voltages reach values beyond the acceptable range, which is unfavourable for costumers. Elimination of negative voltage phenomena coming from RES' is possible thanks to dedicated and autonomous management and control systems. An example of such a solution has been shown in the article-mastering too large voltage changes is possible not only by means of classic $\mathrm{HV} / \mathrm{MV}$ transformer ratio control, but also by means of power consumption control of 
flexible loads and simultaneous use of the RES possibilities in the scope of reactive power generation or consumption. It has been shown that the application of the installation of electrolyzers in the MV network has a positive impact not only on the optimum voltage values, from the point of view of the applied quality indicator, but also on the reduction of power losses. This is understandable, because the excess power generated in renewable sources is consumed locally, as a result of connecting electrolyzers.

Thus, the opinion about the beneficial impact of the consumption subordinate to remote control (and such may include modular electrolyzers) has been confirmed for the network model under consideration, corresponding to the actual networks.

The new concept of control will not only allow to maintain the voltage value in MV network nodes to the extent required by the regulations, but will also ensure the minimum (i.e., optimal) value of a dedicated indicator of its quality. This has been proven in the calculations performed and described. A significant improvement of the voltage quality index (defined by relation (1)) was found, as well as a visible alignment of the voltage profile, which justifies the advisability of seeking new solutions in the field of voltage control in MV networks, taking into account distributed generation.

The originality of the proposed solution lies in the use of as many as three types of control variables (OLTC tap changer, reactive powers of distributed sources and active powers of selected loads). The effects of the control clearly show the synergy of their joint action in accordance with the original heuristic algorithm for minimization of the objective function. Despite the relatively small number of control variables, the effect of their operation giving a very large improvement in voltage quality would not be possible to obtain intuitively. The authors consider this effect to be the greatest achievement of the article, although they argue that the practical implementation of the proposed system still requires solving a number of engineering problems.

The process of optimal voltage control can take place on condition that the actual network is correctly mapped in the optimization process. The creation of a calculation model involves the estimation of the state of MV networks, which is possible due to the current and voltage measurements performed. The radial structure of the network, a small number of nodes, reduces the calculation complexity of this problem, compared to multi-node closed HV networks. It seems that the implementation of the proposed voltage control system is possible in practice, due to the small size of the calculation task and, consequently, the short time of obtaining results, enabling the process of optimal control in real time.

Author Contributions: Conceptualization, P.K. and P.P.; methodology, P.K. and P.P.; software, P.K. and P.P.; validation, P.K. and P.P.; formal analysis, P.K. and P.P.; investigation, P.K. and P.P.; writingoriginal draft preparation, P.K. and P.P.; writing - review and editing, P.K. and P.P.; visualization, P.P.; supervision, P.K. All authors have read and agreed to the published version of the manuscript.

Funding: This research received no external funding.

Institutional Review Board Statement: Not applicable.

Informed Consent Statement: Not applicable.

Data Availability Statement: Not applicable.

Conflicts of Interest: The authors declare no conflict of interest.

\section{References}

1. Farkas, C.; Tóth, A.; Orlay, I. Voltage Control Methods in the MV Grid with a Large Share of PV. Int. J. Emerg. Electr. Power Syst. 2019, 20. [CrossRef]

2. Sigalo, M.B.; Eze, O.K.; Usman, R. Analysis of medium and low voltage distribution network with high level penetration of distributed generators using eracs. Eur. J. Eng. Technol. 2016, 4, 9-23.

3. Kacejko, P.; Adamek, S.; Wydra, M. Optimal voltage control in distribution networks with dispersed generation. In Proceedings of the 2010 IEEE PES Innovative Smart Grid Technologies Conference Europe (ISGT Europe), Gothenburg, Sweden, 11-13 October 2010; IEEE: Piscataway, NJ, USA, 2010; pp. 1-4, ISBN 978-1-4244-8508-6. 
4. Kacejko, P.; Pijarski, P. Zarządzanie mikroinstalacjami OZE—Realne wyzwanie techniczne, czy tylko impuls marketingowy? [Mangement of microgenerations of renewable energy sources-Technical chalange or the marketing impuls?]. Rynek Energii 2016, 2016, 41-45.

5. Zhang, D.; Li, J.; Hui, D. Coordinated control for voltage regulation of distribution network voltage regulation by distributed energy storage systems. Prot. Control Mod. Power Syst. 2018, 3, 3. [CrossRef]

6. Adamek, S. Optimal Voltage Control in Distributed Power Grid with Dispersed Generation. Ph.D. Dissertation, Lublin University of Technology, Lublin, Poland, 2010.

7. Kot, A. Optimal Voltage Control in Medium Voltage Distribution Networks with Dispersed Generation. Ph.D. Dissertation, AGH University of Science and Technology, Cracow, Poland, 2005.

8. European Commission. Communication from the Commission to the European Parliament, the Council, the European Economic and Social Committee and the Committee of the Regions: A Hydrogen Strategy for a Climate-Neutral Europe. COM(2020) 301; European Commission: Brussels, Belgium, 2020.

9. Brauns, J.; Turek, T. Alkaline Water Electrolysis Powered by Renewable Energy: A Review. Processes 2020, 8, 248. [CrossRef]

10. Gutiérrez-Martín, F.; Ochoa-Mendoza, A.; Rodríguez-Antón, L.M. Pre-investigation of water electrolysis for flexible energy storage at large scales: The case of the Spanish power system. Int. J. Hydrogen Energy 2015, 40, 5544-5551. [CrossRef]

11. Hashimoto, K.; Yamasaki, M.; Fujimura, K.; Matsui, T.; Izumiya, K.; Komori, M.; El-Moneim, A.A.; Akiyama, E.; Habazaki, H.; Kumagai, N.; et al. Global CO2 recycling-novel materials and prospect for prevention of global warming and abundant energy supply. Mater. Sci. Eng. A 1999, 267, 200-206. [CrossRef]

12. Hashimoto, K.; Kumagai, N.; Izumiya, K.; Takano, H.; Kato, Z. The production of renewable energy in the form of methane using electrolytic hydrogen generation. Energy Sustain. Soc. 2014, 4, 17. [CrossRef]

13. Götz, M.; Lefebvre, J.; Mörs, F.; McDaniel Koch, A.; Graf, F.; Bajohr, S.; Reimert, R.; Kolb, T. Renewable Power-to-Gas: A technological and economic review. Renew. Energy 2016, 85, 1371-1390. [CrossRef]

14. Eveloy, V.; Gebreegziabher, T. A Review of Projected Power-to-Gas Deployment Scenarios. Energies 2018, 11, 1824. [CrossRef]

15. Akinyele, D.O.; Rayudu, R.K. Review of energy storage technologies for sustainable power networks. Sustain. Energy Technol. Assess. 2014, 8, 74-91. [CrossRef]

16. Sarrias-Mena, R.; Fernández-Ramírez, L.M.; García-Vázquez, C.A.; Jurado, F. Electrolyzer models for hydrogen production from wind energy systems. Int. J. Hydrogen Energy 2015, 40, 2927-2938. [CrossRef]

17. Belderbos, A.; Delarue, E.; D'haeseleer, W. Possible role of power-to-gas in future energy systems. In Proceedings of the 201512 th International Conference on the European Energy Market (EEM), Lisbon, Portugal, 19-22 May 2015; IEEE: Piscataway, NJ, USA, 2015; pp. 1-5, ISBN 978-1-4673-6692-2.

18. Mazza, A.; Bompard, E.; Chicco, G. Applications of power to gas technologies in emerging electrical systems. Renew. Sustain. Energy Rev. 2018, 92, 794-806. [CrossRef]

19. Jentsch, M.; Trost, T.; Sterner, M. Optimal Use of Power-to-Gas Energy Storage Systems in an 85\% Renewable Energy Scenario. Energy Procedia 2014, 46, 254-261. [CrossRef]

20. Park, C.; Bigler, F.; Korba, P. Power-to-Gas Concept for Integration of Increased Photovoltaic Generation into the Distribution. Energy Procedia 2016, 99, 411-417. [CrossRef]

21. Pleßmann, G.; Erdmann, M.; Hlusiak, M.; Breyer, C. Global Energy Storage Demand for a 100\% Renewable Electricity Supply. Energy Procedia 2014, 46, 22-31. [CrossRef]

22. Johnson, J.X.; de Kleine, R.; Keoleian, G.A. Assessment of energy storage for transmission-constrained wind. Appl. Energy 2014, 124, 377-388. [CrossRef]

23. Bailera, M.; Lisbona, P.; Romeo, L.M.; Espatolero, S. Power to Gas projects review: Lab, pilot and demo plants for storing renewable energy and $\mathrm{CO}_{2}$. Renew. Sustain. Energy Rev. 2017, 69, 292-312. [CrossRef]

24. Gahleitner, G. Hydrogen from renewable electricity: An international review of power-to-gas pilot plants for stationary applications. Int. J. Hydrogen Energy 2013, 38, 2039-2061. [CrossRef]

25. Mazza, A.; Salomone, F.; Arrigo, F.; Bensaid, S.; Bompard, E.; Chicco, G. Impact of Power-to-Gas on distribution systems with large renewable energy penetration. Energy Convers. Manag. X 2020, 7, 100053. [CrossRef]

26. Grueger, F.; Möhrke, F.; Robinius, M.; Stolten, D. Early power to gas applications: Reducing wind farm forecast errors and providing secondary control reserve. Appl. Energy 2017, 192, 551-562. [CrossRef]

27. Simonis, B.; Newborough, M. Sizing and operating power-to-gas systems to absorb excess renewable electricity. Int. J. Hydrogen Energy 2017, 42, 21635-21647. [CrossRef]

28. Beccali, M.; Brunone, S.; Finocchiaro, P.; Galletto, J.M. Method for size optimisation of large wind-hydrogen systems with high penetration on power grids. Appl. Energy 2013, 102, 534-544. [CrossRef]

29. Sawas, A.; Farag, H.E.Z. Optimal Sizing of Power-to-Gas Units toward Elevated Renewable Power Penetration. In Proceedings of the 2019 IEEE Canadian Conference of Electrical and Computer Engineering (CCECE), Edmonton, AB, Canada, 5-8 May 2019; IEEE: Piscataway, NJ, USA, 2019; pp. 1-4, ISBN 978-1-7281-0319-8.

30. Diaz de Cerio Mendaza, I.; Bhattarai, B.P.; Kouzelis, K.; Pillai, J.R.; Bak-Jensen, B.; Jensen, A. Optimal sizing and placement of power-to-gas systems in future active distribution networks. In Proceedings of the 2015 IEEE Innovative Smart Grid TechnologiesAsia (ISGT ASIA), Bangkok, Thailand, 3-6 November 2015; IEEE: Piscataway, NJ, USA, 2015; pp. 1-6, ISBN 978-1-5090-1238-1. 
31. Robinius, M.; Raje, T.; Nykamp, S.; Rott, T.; Müller, M.; Grube, T.; Katzenbach, B.; Küppers, S.; Stolten, D. Power-to-Gas: Electrolyzers as an alternative to network expansion-An example from a distribution system operator. Appl. Energy 2018, 210, 182-197. [CrossRef]

32. Buttler, A.; Spliethoff, H. Current status of water electrolysis for energy storage, grid balancing and sector coupling via power-togas and power-to-liquids: A review. Renew. Sustain. Energy Rev. 2018, 82, 2440-2454. [CrossRef]

33. Liu, X.; Aichhorn, A.; Liu, L.; Li, H. Coordinated Control of Distributed Energy Storage System With Tap Changer Transformers for Voltage Rise Mitigation Under High Photovoltaic Penetration. IEEE Trans. Smart Grid 2012, 3, 897-906. [CrossRef]

34. Reihani, E.; Sepasi, S.; Roose, L.R.; Matsuura, M. Energy management at the distribution grid using a Battery Energy Storage System (BESS). Int. J. Electr. Power Energy Syst. 2016, 77, 337-344. [CrossRef]

35. Teng, J.-H.; Luan, S.-W.; Lee, D.-J.; Huang, Y.-Q. Optimal Charging/Discharging Scheduling of Battery Storage Systems for Distribution Systems Interconnected With Sizeable PV Generation Systems. IEEE Trans. Power Syst. 2013, 28, 1425-1433. [CrossRef]

36. El-Taweel, N.A.; Khani, H.; Farag, H.E.Z. Voltage regulation in active power distribution systems integrated with natural gas grids using distributed electric and gas energy resources. Int. J. Electr. Power Energy Syst. 2019, 106, 561-571. [CrossRef]

37. Dalmau, A.R.; Perez, D.M.; Diaz de Cerio Mendaza, I.; Pillai, J.R. Decentralized voltage control coordination of on-load tap changer transformers, distributed generation units and flexible loads. In Proceedings of the 2015 IEEE Innovative Smart Grid Technologies-Asia (ISGT ASIA), Bangkok, Thailand, 3-6 November 2015; IEEE: Piscataway, NJ, USA, 2015; pp. 1-6, ISBN 978-1-5090-1238-1.

38. Han, X.; Kosek, A.M.; Morales Bondy, D.E.; Bindner, H.W.; You, S.; Tackie, D.V.; Mehmedalic, J.; Thordarson, F. Assessment of distribution grid voltage control strategies in view of deployment. In Proceedings of the 2014 IEEE International Workshop on Intelligent Energy Systems (IWIES), San Diego, CA, USA, 8 October 2014; IEEE: Piscataway, NJ, USA, 2014; pp. 46-51, ISBN 978-1-4799-5857-3.

39. Estermann, T.; Newborough, M.; Sterner, M. Power-to-gas systems for absorbing excess solar power in electricity distribution networks. Int. J. Hydrogen Energy 2016, 41, 13950-13959. [CrossRef]

40. Artale, G.; Caravello, G.; Cataliotti, A.; Cosentino, V.; Di Cara, D.; Guaiana, S.; Nguyen Quang, N.; Palmeri, M.; Panzavecchia, N.; Tinè, G. A Virtual Tool for Load Flow Analysis in a Micro-Grid. Energies 2020, 13, 3173. [CrossRef]

41. Coletta, G.; Laso, A.; Jonsdottir, G.M.; Manana, M.; Villacci, D.; Vaccaro, A.; Milano, F. On-Line Control of DERs to Enhance the Dynamic Thermal Rating of Transmission Lines. IEEE Trans. Sustain. Energy 2020, 11, 2836-2844. [CrossRef]

42. Jean Philippe, W.P.; Eftekharnejad, S.; Ghosh, P.K. Development of a new voltage stability index and its implementation considering voltage measurement uncertainty. IET Gener. Transm. Distrib. 2019, 13, 4011-4020. [CrossRef]

43. IEC 60038: IEC Standard Voltages; International Electrotechnical Commission (IEC): Geneva, Switzerland, 2009.

44. EN 50160: Voltage Characteristics of Electricity Supplied by Public Distribution Network; PKN: Warsaw, Poland, 2019.

45. Dib, M.; Ramzi, M.; Nejmi, A. Voltage regulation in the medium voltage distribution grid in the presence of renewable energy sources. Mater. Today Proc. 2019, 13, 739-745. [CrossRef]

46. Guo, Y.; Wu, Q.; Gao, H.; Shen, F. Distributed voltage regulation of smart distribution networks: Consensus-based information synchronization and distributed model predictive control scheme. Int. J. Electr. Power Energy Syst. 2019, 111, 58-65. [CrossRef]

47. Małkowski, R.; Izdebski, M.; Miller, P. Adaptive Algorithm of a Tap-Changer Controller of the Power Transformer Supplying the Radial Network Reducing the Risk of Voltage Collapse. Energies 2020, 13, 5403. [CrossRef]

48. Murty, P.S.R. Power Systems Analysis; Elsevier Science: Saint Louis, MO, USA, 2017; ISBN 9780081011119.

49. Salam, M.A. Fundamentals of Electrical Power Systems Analysis; Springer: Singapore, 2020; ISBN 978-981-15-3211-5.

50. Zhu, J. Optimization of Power System Operation, 2nd ed.; Wiley: Hoboken, NJ, USA; IEEE Press: Piscataway, NJ, USA, 2015; ISBN 9781118887004.

51. Ebeed, M.; Kamel, S.; Jurado, F. Optimal Power Flow Using Recent Optimization Techniques. In Classical and Recent Aspects of Power System Optimization; Elsevier: Amsterdam, The Netherlands, 2018; pp. 157-183. ISBN 9780128124413.

52. Glavitsch, H.; Bacher, R. Optimal Power Flow Algorithms. In Analysis and Control System Techniques for Electric Power Systems, Part 1 of 4; Elsevier: Amsterdam, The Netherlands, 1991; pp. 135-205. ISBN 9780120127412.

53. Pijarski, P.D. Optymalizacja Heurystyczna w Ocenie Warunków Pracy i Planowaniu Rozwoju Systemu Elektroenergetycznego [Heuristic Optimization in the Assessment of Operating Conditions and Development Planning of the Power System]; Wydawnictwo Politechniki Lubelskiej [Lublin University of Technology Publishers]: Lublin, Poland, 2019; ISBN 978-83-7947-349-6.

54. Kumar, K.; Zindani, D.; Davim, J.P. Optimizing Engineering Problems through Heuristic Techniques; CRC Press: Boca Raton, FL, USA, 2020; ISBN 9781138485365.

55. Pesaran Hajiabbas, M.; Mohammadi-Ivatloo, B. Optimization of Power System Problems. Methods, Algorithms and MATLAB Codes; Springer Nature: Berlin/Heidelberg, Germany, 2020; ISBN 978-3-030-34049-0.

56. Radosavljević, J. Metaheuristic Optimization in Power Engineering; The Institution of Engineering and Technology: London, UK, 2018; ISBN 9781785615467.

57. Swamy, M.N.S. Search and Optimization by Metaheuristics_Techniques and Algorithms Inspired by Nature; Birkhauser Verlag Ag: Basel, Switzerland, 2016; ISBN 978-3-319-41191-0.

58. Bozorg-Haddad, O.; Solgi, M.; Loaiciga, H.A. Meta-Heuristic and Evolutionary Algorithms for Engineering Optimization; Wiley Blackwell: Chichester, UK, 2017; ISBN 978-1-119-38699-5. 
59. Pijarski, P.; Kacejko, P. A new metaheuristic optimization method: The algorithm of the innovative gunner (AIG). Eng. Optim. 2019, 51, 2049-2068. [CrossRef]

60. Mirjalili, S.; Mirjalili, S.M.; Lewis, A. Grey Wolf Optimizer. Adv. Eng. Softw. 2014, 69, 46-61. [CrossRef]

61. Pham, D.T.; Ghanbarzadeh, A.; Koç, E.; Otri, S.; Rahim, S.; Zaidi, M. The Bees Algorithm-A Novel Tool for Complex Optimisation Problems. In Intelligent Production Machines and Systems; Elsevier: Amsterdam, The Netherlands, 2006; pp. 454-459. ISBN 9780080451572.

62. Kennedy, J.; Eberhart, R. Particle swarm optimization. In Proceedings of the ICNN'95-International Conference on Neural Networks, Perth, WA, Australia, 27 November-1 December 1995; IEEE: Piscataway, NJ, USA, 1995; pp. 1942-1948, ISBN 0-7803-2768-3.

63. Mirjalili, S. Moth-flame optimization algorithm: A novel nature-inspired heuristic paradigm. Knowl. Based Syst. 2015, 89, $228-249$. [CrossRef]

64. Valsera-Naranjo, E.; Sumper, A.; Villafafila-Robles, R.; Martínez-Vicente, D. Probabilistic Method to Assess the Impact of Charging of Electric Vehicles on Distribution Grids. Energies 2012, 5, 1503-1531. [CrossRef]

65. Yang, X.-S.; Deb, S. Cuckoo Search via Lévy flights. In Proceedings of the 2009 World Congress on Nature \& Biologically Inspired Computing (NaBIC), Coimbatore, India, 9-11 December 2009; IEEE: Piscataway, NJ, USA, 2009; pp. 210-214, ISBN 978-1-4244-5053-4. 